\title{
5th
}

\section{Combinatorial Chemistry} Yields High-temperature Bulk Metallic Glasses

\author{
By YAN Fusheng (Staff Reporter)
}

M etallic glasses, also known as amorphous or glassy alloys, are considered to be the nextgeneration materials following steel and plastic. the two materials essential to so much of our infrastructure and technology. Because metallic glasses can combine the edges of steel and plastic - They are strong, shapeable and corrosion resistant; like metals, they conduct heat and electricity. These properties make them ideal for fashioning gears, precision molds and electrochemical devices, as well as key parts in many high-tech fields such as aerospace and national defense.

Since their discovery in 1960, metallic glasses based on a wider range of elements have been developed. Though many criteria have been proposed for the selection of glass-forming compositions - alloy compositions that would favor glass formation. none works universally. The discovery of glassy alloys with specific properties has so far largely been the result of trial and error. Such trial-and-error approaches are very time-consuming and low productive. The difficulty of the acts of this treasure hunting is comparable to looking for a needle in a haystack. New methods to screen glassy alloys with expected properties in a short time are highly demanded.

To meet this need, a joint team led by Prof. LIL Yanhui, a materials scientist from the CAS Institute of Physics (IOP), resorted to combinatorial chemistry and rapidly identified a new class of metallic glasses that could work well at high temperatures and are easy to be processed into useful components.

They chose iridium (Ir), tantalum (Ta) and nickel

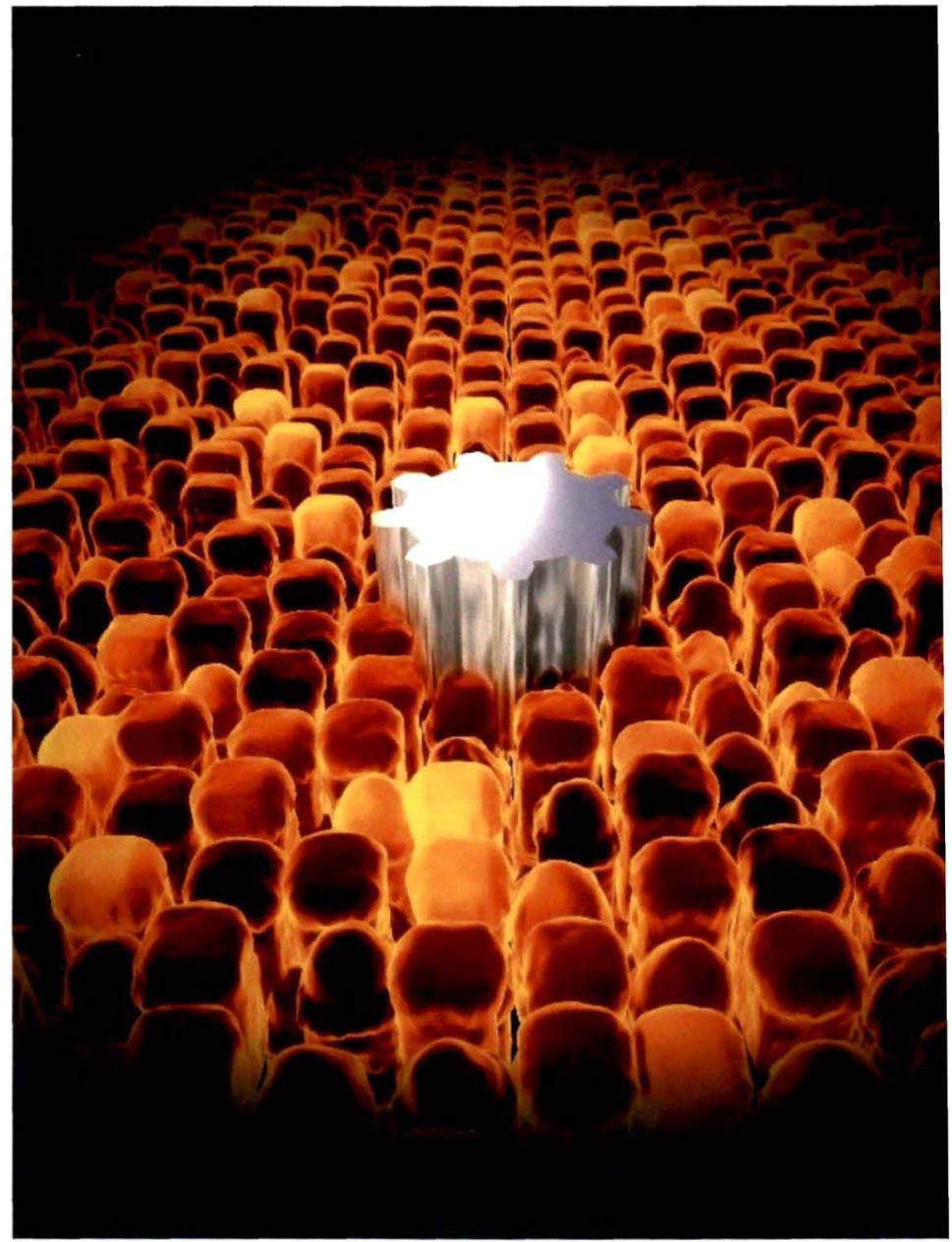

Using combinatorial chemistry, scientists established a high-throughput way to make a new class of high-temperature bulk metallic glasses by screening over a thousand of alloy candidates that vary slightly in chemical compositions on single silicon wafer. (Credit: IOP) 
(Ni), and sputtered them onto a silicon wafer to make a library of thousands of alloys, each slightly differs in the ratios of the three metals. To identify alloys of interest, they used a simplified combinatorial approach harnessing a previously reported correlation between glass-forming ability (GFA) and electrical resistivity. Using this correlation as a guideline, they could rapidly narrow down the optimal composition range that favor the glass formation within two hours. They found that within the composition range of $20 \sim 35 \%$ Ir, $35 \sim 40 \%$ Ta, and $25 \sim 40 \%$ Ni, 2-mm-diameter fully glassy rods are readily achievable. They found the addition of boron further improves CFA, enabling glassy rods with larger cross-section to be made. They also demonstrated that these glassy rods can be easily processed into nanowires and mini-gears.

These new materials have a glass transition temperature of up to 1,162 kelvin, which means that they can withstand much heat without losing their mechanical and physicochemical properties. Notably, they have a wide supercooled liquid region (a temperature window between glass transition and crystallization) up to 136 kelvin, which is wider than that of most existing metallic glasses. Such wide liquid window allows a great deal of thermoplastic formability. and greatly simplifies the practical production of these materials. They can also withstand extremely corrosive conditions: rods made with them showed no weight loss for up to 112 days in aqua regia (mixture of concentrated nitric and hydrochloric acids). while those made of zirconium-based and iron-based metallic glasses were totally corroded in less than an hour.

Over 60 years of global efforts, only a dozen of glassy alloys have marched into practical applications. It is conceivable that the discoveries represent only the tip of an iceberg. There are much more below the surface waiting to be revealed.

The practicality of their design and discovery approach, exemplified by the identification of highstrength, high-temperature bulk metallic glasses, heralds a more efficient way to develop other new glassy alloys with exciting properties.

\section{Reference}

Ming-Xing Li, Shao-Fan Zhao, Zhen Lu, Akihiko Hirata, Ping Wen, Hai-Yang Bai, MingWei Chen, Jan Schroers, YanHui Liu*, Wei-Hua Wang, (2019) High-temperature bulk metallic glasses developed by combinatorial methods. Nature 569, 99. doi: 10.1038/s41586-019-1145-z. 